\title{
PROGRAMAS E RESPOSTAS À SAÙDE DO IDOSO EM PORTUGAL
}

\author{
Dora Margarida Ribeiro Machado \\ ACES Grande Porto III Maia/Valongo, USF Pirâmides dora.ribeiromachado@gmail.com \\ Maria Fernanda Queirós da Mota \\ ACES Tâmega II, Vale de Sousa Sul. USF S. Martinho \\ Manuel Alberto Morais Brás \\ Instituto Politécnico de Bragança, Investigador Integrado no CINTESIS e Professor na Escola Superior de \\ Saúde de Bragança, Portugal. \\ Eugénia Maria Garcia Jorge Anes \\ Instituto Politécnico de Bragança, Investigador Integrado da UICISA: e Professora na Escola de Saúde IPB. \\ Bragança, Portugal.
}

\section{RESUMO}

0 processo de envelhecimento inicia-se à nascença e sabemos que a saúde não é uma questão aleatória, relaciona-se com escolhas, com 0 meio no qual se vive e com 0 acesso à saúde que se dispõe. 0 envelhecimento é diversificado, mas os profissionais tendem a generalizar os cuidados. Verificam-se políticas inadequadas, serviços desajustados, acessibilidade aos cuidados condicionada e consultas de Enfermagem desadequadas. É primordial que se analisem os documentos emanados pelas estruturas da saúde e se reflita numa prática de consulta direcionada para 0 idoso. 0 objetivo deste estudo é abordar a pertinência e aplicabilidade do Programa Nacional para a Saúde das Pessoas Idosas, do Relatório Mundial de Envelhecimento e Saúde, da Estratégia Nacional para o Envelhecimento Ativo e Saudável e da proposta para o Plano Nacional de Saúde para as Demências, apresentando uma reflexão crítica sobre a implicação destas na prática de cuidados ao idoso. Da análise efetuada, concluímos que a tomada de decisão Portuguesa não reflete a implementação de medidas ao mesmo ritmo de outros países da UE. É urgente e pertinente a criação de um programa em exclusivo para as pessoas idosas, que valorize a realização periódica de avaliações dos utentes, permitindo a identificação precoce de alterações e, por outro lado, desenvolva um plano individual de cuidados personalizados a cada um deles. 0 paradigma Biomédico deve ser quebrado sendo essencial que se realize uma Consulta de Enfermagem ao Idoso estruturada, com recurso à avaliação de cinco vertentes: avaliação clínica cuidada; avaliação física; avaliação mental; avaliação 


\section{PROGRAMAS E RESPOSTAS À SAÙDE DO IDOSO EM PORTUGAL}

funcional e; avaliação social. Na tomada de decisão, deve ser primordial a implementação de programas aos vários níveis de prevenção, no sentido de promover o estado de saúde e melhoria da qualidade de vida dos idosos.

Palavras chave: idoso; saúde da família; planos e programas de saúde; demência

\section{ABSTRACT}

Programs and responses to the health of the elderly in Portugal. The aging process starts at birth and we know that health is not a random issue, it is related to choices, the environment in which we live and the access to health that we have. Aging is diverse, but professionals tend to generalize care. There are inadequate policies, inadequate services, accessibility to conditioned care and inadequate nursing consultations. It is essential to analyze the documents issued by health structures and reflect on a practice of consultation directed at the elderly. The objective of this study is to address the pertinence and applicability of the National Program for the Health of Older Persons, the World Report on Aging and Health, the National Strategy for Active and Healthy Aging and the proposal for the National Health Plan for Dementia, presenting a critical reflection on their implication in the care of the elderly. From the analysis carried out, we conclude that Portuguese decisionmaking does not reflect the implementation of measures at the same pace as other EU countries. It is urgent and pertinent to create a program exclusively for the elderly, which values the periodic evaluation of users, allowing the early identification of changes and, on the other hand, develop an individual personalized care plan for each one. The Biomedical paradigm must be broken, and it is essential to carry out a structured Nursing Consultation for the Elderly, using the five-point evaluation: careful clinical evaluation; physical assessment; mental assessment; functional assessment and; social evaluation. In decision-making, the implementation of programs at various levels of prevention must be paramount, in order to promote health status and improve the quality of life of the elderly.

Keywords: aged; family health; health programs and plans; dementia

\section{INTRODUÇÃO}

Em Portugal, a população idosa é cada vez mais envelhecida e, em oposição, a população com menos de 15 anos tem vindo a diminuir. Projeções nacionais apontam para que em 2030 os idosos representem cerca de 26\% da população e em 2060 aumentem para 29\% (FFMS, 2019).

0 envelhecimento é parte constituinte do ciclo vital da pessoa, sendo crucial que seja vivido de forma saudável e autónoma o maior número de anos possível, pelo que os Cuidados de Saúde são decisivos no desenvolvimento de estratégias que promovam a saúde e previnam a doença, nomeadamente nesta fração populacional.

Em termos cronológicos, em 2004 a Direção-Geral da Saúde (DGS) aprovou o Programa Nacional para a Saúde das Pessoas Idosas (PNSPI), em 2015 a Organização Mundial de Saúde (OMS) emitiu o Relatório Mundial de Envelhecimento e Saúde, em 2017 surgiu a Estratégia Nacional para o Envelhecimento Ativo e Saudável (ENEAS) e em 2018 a proposta para o Plano Nacional de Saúde para as Demências.

Face ao exposto, o presente documento tem como objetivo abordar a pertinência e aplicabilidade de cada um dos programas acima referidos e apresentar uma reflexão crítica sobre a prática de cuidados ao idoso. Para fundamentar a reflexão, realizou-se uma pesquisa bibliográfica operacionalizada na SciELO e no Repositório Científico de Acesso Aberto de Portugal (RCAAP), com as seguintes palavras-chave: Idoso, Saúde da Família, Planos e Programas de Saúde, Demência. 


\section{ENQUADRAMENTO TEÓRICO}

A inversão da pirâmide etária constitui um desafio para os governos, sociedade no geral e famílias (FFMS, 2019). É primordial que se reflita no envelhecimento numa atitude de prevenção de doenças e dependência e de promoção da autonomia e saúde. Para que o idoso mantenha a qualidade de vida e assegure um papel ativo na sociedade é essencial que tenha saúde. Contudo, os progressos da medicina têm exponenciado situações de doença e incapacidade.

No intuito de promover a saúde, durante 0 ciclo vital do indivíduo, e prevenir ou retardar situações de doença ou de dependência foram elaborados planos de saúde que visam a "(...) manutenção de um envelhecimento saudável, a promoção da autonomia e a melhoria da intervenção dos prestadores de cuidados" (Martins, 2003, p. 67).

Em 2004, integrado no Plano Nacional de Saúde (PNS) 2004-2010, foi aprovado o PNSPI cuja aplicação deveria ser realizada pelos profissionais da Rede de cuidados de saúde primários, secundários e de cuidados continuados (Ministério da Saúde [MS], 2004). Visava a “(...) manutenção da autonomia, independência, qualidade de vida e recuperação global das pessoas idosas, prioritariamente no seu domicílio (...)", definindo estratégias de intervenção que objetivavam ganhos de saúde e uma melhoria das "(...) práticas profissionais no âmbito das especificidades do envelhecimento (...)". Para tal, promovia um envelhecimento ativo, com a adequação dos cuidados às necessidades das pessoas idosas e com a promoção de ambientes capacitadores, defendendo que "(...) políticas que permitam desenvolver ações mais próximas dos cidadãos idosos, capacitadoras da sua autonomia e independência (...) permitem minimizar custos, evitar dependências, humanizar os cuidados e ajustar-se à diversidade que caracteriza o envelhecimento" (MS, 2004).

Em 2015 a OMS emitiu um Relatório Mundial de Envelhecimento e Saúde, onde ficou evidenciado que a longevidade não se deve apenas ao fator genético, mas também aos ambientes físico e social, nos quais as pessoas se inserem. No mesmo documento, alertou para a forma estereotipada como é encarada a idade do indivíduo que, não raras vezes, cria uma discriminação etária podendo levar a uma ênfase na contenção de custos, por suposição que os gastos com idosos são um dreno para a economia. Aliás, esta organização defende que as pessoas mais velhas contribuem de muitas formas para os recursos da sociedade onde se inserem, nomeadamente como forma de apoio emocional, pelo que a política dos países deve ser adaptada para que se promova a capacidade dos idosos para realizar essas múltiplas contribuições. Defende igualmente que as políticas de saúde devem ter em conta as mudanças sociais e tecnológicas que a sociedade vai experienciando, já que 0 custo associado ao envelhecimento está diretamente ligado às mesmas e, per si, apenas a um incremento de $2 \%$. Com base nestes pressupostos a OMS definiu um novo conceito para 0 envelhecimento saudável, apresentando-o como "(...) o processo de desenvolvimento e manutenção da capacidade funcional que permite 0 bem-estar em idade avançada" e defendeu a necessidade de uma ação urgente de saúde pública direcionada para 0 envelhecimento com 0 objetivo de maximizar a capacidade funcional do indivíduo. Neste sentido, emitiu diretrizes para gerir declínios na capacidade intrínseca, divididas em seis módulos: (i) melhorar a função musculosquelética, a mobilidade e a vitalidade; (ii) manter as funções sensoriais; (iii) prevenir a deterioração cognitiva grave e promover o bem-estar psicológico; (iv) fazer frente a problemas associados à idade, como a incontinência urinária; prevenir as quedas; e (vi) apoiar os cuidadores (OMS, 2015, p. 13).

A ENEAS surge em 2017, no âmbito dos programas de promoção de saúde e prevenção de doença e do PNS, prevendo a promoção de vidas saudáveis e bem-estar de todos, independentemente da sua idade. Tem como premissa a individualidade e diversidade do processo de envelhecimento, defendendo medidas no âmbito da promoção de estilos de vida saudáveis e vigilância da saúde e da gestão dos processos de comorbilidade. Desta forma, demanda que se maximize a capa- 


\section{PROGRAMAS E RESPOSTAS À SAÙDE DO IDOSO EM PORTUGAL}

cidade funcional das pessoas idosas, para que as mesmas possam continuar o seu desenvolvimento e o seu papel ativo na sociedade nos cinco domínios: satisfação das necessidades; desenvolvimento e tomada de decisão informada; capacidade de aprendizagem; capacidade de contribuição para as suas famílias e comunidades; capacidade para fazer e manter relacionamentos; capacidade para se movimentarem (DGS, 2017a). Neste documento é também explorada a importância de serem asseguradas medidas de segurança para as pessoas idosas, nomeadamente através da promoção de ações de sensibilização no âmbito da prevenção/proteção de situações de crime e na orientação de idosos e cuidadores para a utilização de tecnologias que promovam a segurança.

Por fim, tendo em conta que em Portugal cerca de 150.000 pessoas sofrem de demência, a maioria das quais com mais de 65 anos sendo que, o principal risco para o seu desenvolvimento é a idade, em 2018 é aprovada a Estratégia de Saúde na Área das Demências. Este documento emite o dever de serem concluídos Planos Regionais da Saúde para as Demências, que devem propor um percurso de Cuidados para a pessoa com demência que se inicie com a identificação precoce da mesma, seguida de um diagnóstico integrado, planeamento de cuidados e intervenção terapêutica. Neste processo não será excluída a capacitação de cuidadores formais e informais (Despacho n.. $5998 / 2018,2018$ ).

Ainda no âmbito da Saúde do Idoso encontram-se Programas e Instrumentos de Saúde que, direta ou indiretamente, beneficiam o mesmo. Destacam-se:

a Rede Nacional de Cuidados Continuados Integrados (RNCCI), criada a partir do Decreto-Lei no 101/2006, destinada à população em situação de dependência, sendo na sua maioria idosa (Sistema Nacional de Saúde [SNS], 2017). Promove a manutenção das pessoas, no seu meio habitual, procurando melhorar a equidade do acesso aos cuidados de saúde de longa duração, transitórios, de qualidade ou flexíveis, através da apresentação de diferentes respostas e níveis de prestação de apoio social e de cuidados de saúde, assegurando ganhos em anos de vida com independência (MS, 2004, p. 12);

o Projeto de Saúde Oral nas Pessoas Idosas (SOPI), integrado no Programa Nacional de Promoção da Saúde Oral (PNPSO) em 2008, após revisão e reestruturação do mesmo. Visa a promoção do tratamento oral, a diminuição e a prevalência de doenças orais, considerando a importância que as mesmas representam na saúde e qualidade de vida das pessoas. Permite o pagamento, através de "cheques dentista", personalizados e atribuídos ao utente idoso na Unidade Funcional onde se encontra inscrito, de cuidados preventivos e curativos de medicina dentária (MS, 2008);

o Programa Nacional de Prevenção de Acidentes, que surge em 2010, articulado com outros programas de saúde, tais como PNS e PNSPI, e tem como principal objetivo a promoção da segurança e a prevenção de acidentes não intencionais, tendo também como população alvo os grupos vulneráveis. Desenvolve-se em oito eixos estratégicos: (i) capacitar os profissionais; (ii) reforçar a ação comunitária; (iii) reforçar a ação intersectorial; (iv) orientar a intervenção dos serviços de saúde; (v) legislar; (vi) monitorizar acidentes não intencionais; (vii) apoiar a investigação; e (viii) cooperar com organizações internacionais (DGS, 2010);

o Programa Nacional para a Promoção da Alimentação Saudável (PNPAS), considerado como um plano de saúde prioritário desde 2012 e que visa capacitar os cidadãos para a tomada de decisão informada na sua alimentação, nomeadamente através da elaboração e divulgação de diferentes documentos;

o Programa Nacional de Educação para a Saúde, Literacia e Autocuidado (PNESLA) que visa contribuir para a melhoria da educação para a saúde, literacia e autocuidado da população, por forma a autonomizar e responsabilizar os cidadãos no seu processo de saúde e dos que deles dependem (Despacho n.. 3618-A/2016); 
o Programa Nacional para a Promoção da Atividade Física (PNPAF) visou universalizar o conceito de estilo de vida fisicamente ativo, independentemente das condições sociodemográficas ou económicas, reduzindo o tempo de sedentarismo. Para tal, procurou aumentar a literacia, valorização e participação da população em diferentes formas de atividade física, capacitar os profissionais de saúde para a promoção da atividade física e promover a vigilância da atividade física e dos seus determinantes (DGS, 2017b);

a Tabela Nacional de Funcionalidade (TNF), elaborada pela DGS, que tem como objetivo adotar políticas de saúde e sociais mediante a funcionalidade da pessoa com doença crónica e não apenas de acordo com a sua incapacidade. A sua aplicabilidade pretende "(...) dotar os profissionais de saúde de informação que permita quantificar o grau de funcionalidade e medir os ganhos de saúde obtidos após intervenção terapêutica (...) e planear as intervenções em saúde (...) de acordo com a funcionalidade da pessoa, melhorando a equidade na atribuição de benefícios de carácter especial a pessoas com doença crónica, incapacidade ou invalidez.". Foi definido que a TNF deverá ser aplicada, no início e no fim do plano de cuidados aplicados, a todas as pessoas com mais de 18 anos de idade, que se apresentem com doença crónica ou com incapacidade permanente/temporária, (MS, 2019, p. 4);

o Plano de Ação para a Literacia em Saúde (2019-2021) que pretende melhorar o nível de literacia da população residente em Portugal, inserindo-a o centro da intervenção. Este plano apresenta uma abordagem por ciclo de vida, enquadrando a sua realização por contexto, setting e objetivos estratégicos (DGS, 2019a);

e o Manual de Boas Práticas Literacia em Saúde - Capacitação dos Profissionais de Saúde, que procura uniformizar e organizar as ações e medidas relacionadas com a Literacia em Saúde, promovendo 0 aumento da Literacia em Saúde da população Portuguesa. Este documento serve como guia para os profissionais de saúde permitindo um maior conhecimento dobre modelos de mudança comportamental (DGS, 2019b).

\section{REFLEXÃO CRÍTICA}

É consensual a importância atribuída ao processo de envelhecimento para que se assegurem a saúde e a qualidade de vida do indivíduo, família e sociedade. Não obstante, as medidas realizadas ficam aquém do necessário.

Apesar da existência do PNSPI e da ENEAS “(...) não se encontra instituída nenhuma metodologia sistematizada para a avaliação gerontológica ou geriátrica das pessoas idosas nos Cuidados de Saúde Primários" (Balsinha \& Pereira, 2014, p. 196).

A estratégia da Saúde na área das Demências, ainda que publicada em 2018, carece de relatórios da sua aplicabilidade. E, na prática, há pouca formação na área, nomeadamente sobre escalas de avaliação cognitiva e aplicabilidade das mesmas, para deteção precoce de demência. Desta forma, os cuidados aplicados ao utente idoso reportam-se ao básico do aplicável na doença aguda ou crónica já instalada, o que gera, não raras vezes, internamentos evitáveis, desperdício de recursos, com consequente aparecimento de dependências, e cansaço dos familiares, que não encontram respostas de suporte.

A RNCCI, embora de extrema importância, revela um número de vagas insuficientes, com um longo tempo de espera, potenciando a fase de declínio em que a pessoa se encontra e diminuindo a possibilidade de recuperação da mesma.

Idosos com maior literacia potenciam a sua saúde, pelo que a literacia prevista pela ENEAS necessita de maior implicação dos diferentes setores. Foi emitido o relatório do PNPAS que objetiva para os anos 2019-2020 a promoção da literacia alimentar e nutricional da população, com a publicação de documentos destinados a profissionais, no entanto pouco se tem divulgado. Também 


\section{PROGRAMAS E RESPOSTAS À SAÙDE DO IDOSO EM PORTUGAL}

foi emitido o relatório do PNPAF, onde fica evidenciada a importância do aconselhamento breve para a atividade física nas consultas aos utentes, carece, contudo, da elaboração de uma norma Clínica e a implementação da consulta de atividade física ainda se encontra em fase experimental. Porém, algumas Câmaras e Juntas de Freguesia disponibilizam acesso gratuito a atividades físicas a utentes idosos, facilitando a acessibilidade a estes cuidados. Não obstante da sua importância, esta medida corre o risco de se revelar segregacionista, por evidenciar uma separação etária inter e intrageracional (Rosas, 2015).

No âmbito da saúde do idoso não existe um programa de consulta de Enfermagem estruturado, ao contrário do que se verifica em outras consultas. Assim, esta consulta baseia- se na promoção e educação para a saúde, cumprimento do Plano Nacional de Vacinação (PNV), tratamento de problemas relacionados com as situações agudas e crónicas e minimização de danos, referenciando para equipas diferenciadas (RNCCI, consultas hospitalares, etc.). No entanto, não é dado ênfase à identificação de grupos de risco e ao diagnóstico precoce de situações que promovam o declínio do idoso, negligenciando os Gigantes da Geriatria, ou sete "is", (1. Incapacidade cognitiva; 2. latrogenia; 3. Incontinência; 4. Instabilidade postural; 5. Imobilidade; 6 . Incapacidade comunicativa; 7. Insuficiência familiar) e condicionando a atuação atempada dos profissionais (Moraes, Marino \& Santos, 2010).

Há falta de formação dos profissionais de saúde no cuidado gerontogeriátrico e baixo número de indicadores de avaliação significativos para a produção de ganhos em saúde nos idosos. No entanto, alguns indicadores centram-se na área da doença crónica que se sabe atinge maioritariamente utentes idosos, pelo que, ainda que não diretamente associado à idade, garante a prestação de cuidados a estes utentes. Além disso, o facto da grelha de indicadores se dirigir substancialmente à prestação de cuidados nas crianças e adultos, a médio e longo prazo será possível obter ganhos em saúde nas pessoas idosas.

Outro dos fatores que poderá condicionar a prestação de cuidados de saúde a este grupo, será 0 limite de 15 minutos por consulta, que se percebe ser pouco para as necessidades avaliativas que um utente geriátrico comporta.

Compreende-se, por tudo isto, a necessidade de se realizar uma consulta específica para 0 idoso, onde sejam avaliados o seu estado de dependência (Katz para as atividades básicas de vida e Lawton \& Brody para as atividades instrumentais de vida diária), o seu estado nutricional (através do questionário Mini-Nutricional Assessment), social (escala de Gijon) e de fragilidade (Escala de Fragilidade de Edmonton) e os diferentes domínios da capacidade intrínseca: 1. da Cognição (questionário Mini- Mental State Examination); 2. da Locomoção (escala de classificação funcional da Marcha de Holden); 3. da Vitalidade (SF-36); 4. Sensorial; 5. Psicológico (escala de depressão geriátrica de Yesavage) .

Só através da colheita de dados para uma correta anamnese, da realização de um exame físico e mental, nomeadamente por aplicação de escalas, análise de exames complementares de diagnóstico e avaliação funcional e social do idoso é que será possível fazer uma síntese do seu estado para identificar problemas e prioridades e definir um plano de ação. E só dessa forma se faz Enfermagem voltada para a precisão do diagnóstico, com maior probabilidade de prognóstico correto e, por conseguinte, com diminuição do risco iatrogénico, facilitando condutas preventivas, orientando intervenções, adequando medidas assistenciais, facilitando o acompanhamento do utente geriátrico e potenciando a sua qualidade de vida.

\section{CONCLUSÕES}

0 processo de envelhecimento inicia-se à nascença, contudo, a dada altura incrementa-se com a deterioração funcional do indivíduo. Assim, já que a probabilidade de doença aumenta com a 
idade, os cuidados de saúde devem ser cada vez mais adaptados à mudança demográfica que vivemos.

Os programas de saúde elaborados visam um envelhecimento saudável e a sua aplicabilidade, renovação, conclusão e avaliação é urgente. Não basta reconhecer que é necessário um investimento nas ações que aumentem o número de anos com independência e melhorem as práticas dos profissionais no contexto do envelhecimento, é preciso agir.

É necessário que seja criado um programa em exclusivo para as pessoas idosas, que valorize a realização periódica de avaliações dos utentes, permita a identificação precoce de alterações e, por outro lado, desenvolva um plano individual de cuidados personalizados a cada um deles. Isso implicará a formação dos profissionais de saúde para uma atitude não discriminatória e adequada ao nível de literacia dos utentes idosos e seus cuidadores.

As políticas em saúde devem incluir mais indicadores específicos à saúde do idoso, onde se sinalizem situações de solidão/isolamento, se incentive a adaptação dos serviços, se priorize 0 atendimento à pessoa idosa e se dinamizem ações de formação e capacitação dirigidas aos cuidadores. É necessário que as Unidades de Cuidados de Saúde Primários se voltem para os idosos e, na mesma ótica dos hospitais amigos dos idosos, adotem medidas que reflitam uma elaboração de processo de cuidados adaptados a esta população.

Ainda que visível, a tomada de decisão Portuguesa não se reflete na elaboração de medidas ao mesmo ritmo que 0 vivenciado por outros países da União Europeia. No entanto, as patologias que provocam um percentual de mortalidade maior nos idosos, e com tendência crescente, são passíveis de prevenção, pelo que é emergente uma planificação de cuidados direcionados e adequados à população idosa.

0 paradigma Biomédico deve ser quebrado e é essencial que se realize uma Consulta de Enfermagem ao Idoso estruturada, com recurso à avaliação de cinco vertentes: (i) avaliação clínica cuidada, através de um exame clínico eficaz e análise de exames complementares de diagnóstico; (ii) avaliação física, com recurso à análise da capacidade física, de marcha e de equilíbrio e do estado de nutrição do utente; (iii) avaliação mental, quer da parte cognitiva, quer da parte afetiva; (iv) avaliação funcional, por inferência do seu grau de autonomia e de independência; e (v) avaliação social, através da análise da família, do seu meio, dos seus recursos económicos e da sua rede social.

0 envelhecimento deverá ser encarado como uma oportunidade, já que a população idosa é cada vez mais escolarizada e por isso poderá desempenhar um papel mais ativo na sociedade, seja na transmissão de cultura, seja na criação de produtos direcionados para as necessidades específicas desta população. Por isso, é essencial que se salvaguarde a sua vulnerabilidade.

Na tomada de decisão, deve ser primordial a implementação de programas aos vários níveis de prevenção, efetivos e específicos para os idosos, no sentido de promover o seu estado de saúde e melhoria da qualidade de vida.

\section{REFERÊNCIAS BIBLIOGRÁFICAS}

Balsinha, M. C., \& Pereira, M. G. (2014). A avaliação do paciente idoso em Medicina Geral e Familiar: desafios e oportunidades. Revista Portuguesa Medicina Geral Familiar, 30(3), 196-201.

Despacho n.․ㅜ 3618-A/2016. Diário da república, 2. série (N.- 49 de 10 de março de 2016), 8660(5)8660(6). Retrieved from http://www2.portaldasaude.pt/NR/rdonlyres/4BDA6E5F- 7D29-42D7ACA0-B50BC852A3B3/0/73833787.pdf

Despacho n.․ 5998/2018 (2018). Aprova a Estratégia da Saúde na Área das Demências e determina a constituição e a composição da Coordenação do Plano Nacional da Saúde para as Demências. 


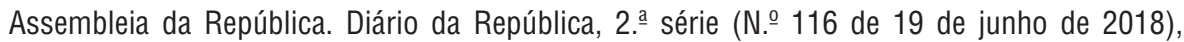
17094-17101. ELI: https://dre.pt/application/conteudo/115533450

Direção-Geral da Saúde (2017a). Estratégia Nacional para o Envelhecimento Ativo e Saudável 20172025. Lisboa: Direção-Geral da Saúde.

Direção-Geral da Saúde (2019b). Manual de Boas Práticas Literacia em Saúde: Capacitação dos Profissionais de Saúde. Lisboa: Direção-Geral da Saúde.

Direção-Geral da Saúde (2019a). Plano de Ação para a Literacia em Saúde 2019-2021 - Portugal. Lisboa: Direção-Geral da Saúde.

Direção-Geral da Saúde (2010). Programa Nacional de Prevenção de Acidentes 2010-2016. Lisboa: Direção-Geral da Saúde.

Direção-Geral da Saúde (2017b). Programa Nacional para a Promoção da Atividade Física 2017. Lisboa: Direção-Geral da Saúde.

FFMS. (2019). Retrato de Portugal [e-book]. Retrieved from https://www.pordata.pt/ebooks/PT2019v20190711/mobile/index.html

Martins, R. (2003). Envelhecimento e saúde: um problema social emergente. Millenium, 6(27), 2542. Retrieved from http://www.ipv.pt/millenium/Millenium27/14.htm

Ministério da Saúde. Direção-Geral da Saúde. Circular Informativa n.ำ 4/DSPPS/DCVAE, de 27 de fevereiro de 2008. Alargamento do Programa Nacional de Promoção da Saúde Oral: Grávidas e Idosos beneficiários do complemento solidário. Lisboa: Direção-Geral da Saúde, 2008.

Ministério da Saúde. Direção-Geral da Saúde. Norma n. o 001, de 25 de janeiro de 2019. Implementação da Tabela Nacional de Funcionalidade no Adulto e Idoso. Lisboa: Direção- Geral da Saúde, 2019.

Ministério da Saúde. Direção-Geral da Saúde. Circular Normativa n.ำ13/DGCG, de 02 de julho de 2004. Programa Nacional para a Saúde das Pessoas Idosas. Lisboa: Direção-Geral da Saúde, 2004.

Moraes, E., Marino, M. \& Santos, R. (2010). Principais síndromes geriátricas, 20(1), 54-66. Retrieved from http://www.observatorionacionaldoidoso.fiocruz.br/biblioteca/_artigos/196.pdf

Organização Mundial da Saúde. (2015). Relatório Mundial de Envelhecimento e Saúde. Suíça: Organização Mundial da Saúde.

Rosas, I. (2015). Idoso, Vulnerabilidade, Risco e Violência: Que medidas de proteção? (Master's thesis, Instituto Superior de Serviço Social do Porto). Retrieved from https://comum.rcaap.pt/bitstream/10400.26/10553/1/Idalina\%20da\%20Conceição\%20Gon çalves\%20Rosas.pdf

Sistema Nacional de Saúde. (2017). Cuidados Continuados Integrados. Retrieved from https://www.sns.gov.pt/sns/reforma-do-sns/cuidados-continuados-integrados-2/ 